\title{
MENTAL HEALTH OUTCOMES OF ADULTS WITH COMORBIDITY AND CHRONIC DISEASES DURING THE COVID-19 PANDEMIC: A MATCHED CASE-CONTROL STUDY
}

\author{
Abu Sayeed ${ }^{1, *}$, Satyajit Kundu ${ }^{2, *}$, Md. Hasan Al Banna ${ }^{3, *}$, Enryka Christopher ${ }^{4}$, M. Tasdik Hasan ${ }^{5}$, \\ Musammet Rasheda Begum ${ }^{6}$, Sukanta Chowdhury ${ }^{7}$ \& Md Shafiqul Islam Khan ${ }^{3}$ \\ ${ }^{1}$ Department of Post-Harvest Technology and Marketing, Patuakhali Science and Technology University, \\ Patuakhali, Bangladesh \\ ${ }^{2}$ Department of Biochemistry and Food Analysis, Patuakhali Science and Technology University, Patuakhali, Bangladesh \\ ${ }^{3}$ Department of Food Microbiology, Patuakhali Science and Technology University, Patuakhali, Bangladesh \\ ${ }^{4}$ Harvard T.H. Chan School of Public Health, Boston, MA, USA \\ ${ }^{5}$ Department of Primary Care and Mental Health, University of Liverpool, Liverpool, UK \\ ${ }^{6}$ Department of Agricultural Economics and Social Sciences, Chattogram Veterinary and Animal Sciences University, \\ Chattogram, Bangladesh \\ ${ }^{7}$ Infectious Diseases Division, International Centre for Diarrhoeal Disease Research, Bangladesh (ICDDR,B),
} Dhaka, Bangladesh

*Authors contributed equally to this manuscript

received: 30.10 .2020 ;

revised: 20.11.2020;

accepted: 1.12 .2020

\section{SUMMARY}

Background: Individuals with certain pre-existing chronic health conditions have been identified as a high-risk group for fatalities of COVID-19. Therefore, it is likely that individuals with chronic diseases may worry during this pandemic to the detriment of their mental health. This study compares the mental health of Bangladeshi adults affected by chronic disease to a healthy, matched control group during the COVID-19 pandemic.

Subjects and methods: A matched case-control analysis was performed with data collected from 395 respondents with chronic diseases and 395 controls matched for age, gender, and residence. Inclusion criteria for cases were respondents who self-reported having asthma, cardiovascular disease symptoms and/or diabetes. Respondents were recruited using an online survey, which included the DASS-21 measure to assess symptoms of stress, anxiety, and depression. Chi-square test, t-test, Fisher's exact test and a conditional logistic regression were performed to examine associations among variables.

Results: The prevalence of anxiety symptoms and depression symptoms and the level of stress were significantly higher among cases $(59 \% ; 71.6 \% ; 73.7 \%$, respectively) than among controls $(25.6 \% ; 31.1 \% ; 43.3 \%$, respectively). Chi-square and t-test showed significant associations and differences between having chronic diseases and mental health outcomes. A conditional logistic regression showed that respondents with asthma, diabetes, cardiovascular disease symptoms, or any combination of these diseases had higher odds of exhibiting symptoms of stress, anxiety, and depression than healthy individuals.

Conclusion: These results underscore a subpopulation vulnerable to mental health consequences during this pandemic and indicate the need for additional mental health resources to be available to those with chronic diseases.

Key words: COVID-19 - mental health - chronic diseases - Bangladesh

\section{INTRODUCTION}

The Coronavirus disease 2019 (COVID-19) pandemic emerged in Wuhan, China and has spread all over the world and has caused huge threats to health and lives (Jakovljevic et al. 2020, Banna et al. 2020), which was then announced as a global pandemic by the WHO in March of 2020 (Marčinko et al. 2020, WHO 2020a). The COVID-19 pandemic has quickly become a worldwide threat, causing substantial morbidity and mortality. The COVID-19 outbreak has induced public and global mental health crisis as well as a huge psychosocial experiment (Jakovljevic et al. 2020).

To date (August 22, 2020), COVID-19 has infected over 23 million people across the world, and almost 800,000 have succumbed to this disease or compli- cations resulting from COVID-19 (WHO 2020b). Particularly, older individuals and those with chronic diseases, such as diabetes, cardiovascular diseases, hypertension, asthma and stroke, seem to be most vulnerable (Onder et al. 2020, Ruan et al. 2020, Yang et al. 2020). Evidence from China and Italy also indicates that the presence of comorbid chronic diseases may increase the risk of death from COVID-19 (Onder et al. 2020, Yang et al. 2020). Extreme quarantine measures and fear have made it difficult for individuals to access routine medical care, especially in low- and middle-income countries managing medicine and human resource shortages (Pellino \& Spinelli 2020). Thus, it is reasonable to anticipate that patients with chronic diseases will face mental health issues such as anxiety, depression, and increased stress (Brooks et al. 2020). 
Existing literature points to a significant association between mental health conditions and chronic diseases, such as diabetes mellitus, cardiovascular diseases and pneumonia (Rozario \& Masho 2018, Mukeshimana \& Chironda 2019). The presence of depressive comorbidity with diabetes in Bangladesh varies from 34\% to $60 \%$ depending on age, income, gender, and diabetes type (Rahman et al. 2011, Roy et al. 2012, Islam et al. $2015 \mathrm{~b}$ ). Amid the uncertainty and disruption of the COVID-19 pandemic in Bangladesh, mental health and chronic disease comorbidity may increase. The COVID19 case fatality rate has been increasing rapidly in Bangladesh (IEDCR 2020). As a result, the mental health of individuals with chronic diseases is impacted by the fear of infection. Compounding deterioration of the mental health of these individuals are factors such as home confinement, limits to outdoor spaces, shortages of essential items, and uncertainty of infection. Along with physical care, mental health care for individuals with chronic diseases must be prioritized. Besides, due to the rapid diffusion of the viral infection, there are already alarms on how to deal with the psychiatric aspects of COVID-19 pandemic in persons with comorbidity and chronic diseases and with an established diagnosis of psychiatric disorders (Lazzari et al. 2020). Thus, understanding how crisis like COVID-19 influence on mental health outcomes of individuals with comorbidity and chronic diseases is important in order to create meaningful and effective interventions (Marčinko et al. 2020).

Considering the context, the authors hypothesized that those with comorbid mental health and chronic diseases in Bangladesh may suffer from mental health issues more than healthy individuals during the pandemic. There is currently no research on psychological distress felt during the COVID-19 pandemic among those with chronic diseases in Bangladesh. This study aims to estimate the prevalence of mental health distress (stress, anxiety, and depression symptoms) among those with and without chronic diseases during the COVID-19 pandemic in Bangladesh.

\section{SUBJECTS AND METHODS}

\section{Study design and Participants}

Health data collected from a web-based, descriptive, cross-sectional survey conducted from April $25^{\text {th }}$ to May $6^{\text {th }}, 2020$ estimated mental health prevalence rates of the general population in Bangladesh. As a community-based survey during this time was not feasible, data was collected online. The authors distributed the survey link in all divisions of Bangladesh via social media such as WhatsApp and Messenger, using snowball sampling. Eligibility criteria included the ability to read Bangla and residence in Bangladesh for the duration of the government mandated lockdown. Of the original 1,427 respondents, 395 reported currently suffering from at least one chronic disease from the survey list (asthma/diabetes/cardiovascular diseases). These respondents with pre-existing chronic diseases were included in the case group for this study. One control was selected for each case and matched for age ( \pm 5 years), sex and area of residence to control for possible confounders. Case and control samples were determined to have similar distributions across those (age, sex and area) variables, which reduced variance in the parameters of interest and improved the study's efficacy (Vandenbroucke et al. 2007, Rose \& Van der Laan 2009, Pearce 2016). Individuals 18 years and older were eligible for the study. Inclusion criteria for the case group was the presence of the following self-reported chronic diseases or symptoms: asthma, diabetes, hypertension, and cardiovascular diseases. Hypertension was considered a cardiovascular disease symptom. Respondents who reported other diseases were excluded, as there were too few to properly match controls to these cases.

\section{Procedures}

The survey questionnaire was first written in English and then translated into Bangla by an expert in both languages. The questionnaire was piloted in a small, randomly selected group of online users to confirm clarity and understanding. The online survey included a short overview of the study context and purpose, eligibility criteria, procedures, consent form and directions on answering the questionnaire. Clicking on the survey link automatically led respondents to the study overview and informed consent page. Demographic information was required to complete the survey and appeared prior to the questionnaire. This study followed the guidance of the Checklist for Reporting Results of Internet E-Surveys (CHERRIES) guidelines (Eysenbach 2004), as well as the 2013 revision of the Helsinki Declaration (Williams 2008).

\section{Survey Questions}

The survey questionnaire comprised 29 close-ended queries, which took four to six minutes to finish. The questionnaire was split into three sections, demographics (six items), physical health (two items) and mental health (21 items). Socio-demographic data were collected on age, gender, education, current residency, monthly income, and smoking history. The two questions on physical health were about any chronic diseases and a selfreport on general physical health status. Mental health was evaluated using a validated Bangla version of the Depression, Anxiety and Stress Scale (DASS-21), with scores measured according to previous research (Le et al. 2019). The DASS-21 includes 21 total self-report items distributed among three factors designed to assess the negative emotional symptoms of depression, anxiety and stress experienced over the past week (Lovibond \& Lovibond 1995). Each item score is on a four-point Likert scale ranging from zero to three, with higher scores indicating a higher frequency or intensity of the item. The total score for depressive symptoms was categorized into normal (0-9), mild depression (10-12), moderate depression (13-20), severe depression (21-27), and extremely severe depression (28-42). The total anxiety subscale 
score was categorized into normal (0-6), mild anxiety $(7$ $9)$, moderate anxiety (10-14), severe anxiety (15-19), and extremely severe anxiety (20-42). Questions 1, 6, 8, $11,12,14$, and 18 formed the stress subscale. The total stress subscale score was categorized into normal (0-10), mild stress (11-18), moderate stress (19-26), severe stress (27-34), and extremely severe stress (35-42) (Banna et al. 2020, Wang et al. 2020). The Bangla DASS-21 has proven to be a reliable and relevant test for the assessment of mental health in the Bangladeshi population (Alim et al. 2015, Sadiq et al. 2019, Khan et al. 2020). Previous research reports a Cronbach's alpha coefficient of 0.82 for the DASS-21, indicating acceptable internal consistency (Taber 2018). Prior to this study, the DASS was used in SARS research (McAlonan et al. 2007).

\section{Statistical Analysis}

Continuous variables, such as the DASS-21 scores, were converted into categorical variables, in order to interpret results by group. Although the nature of this survey is continuous, the decision to categorize scores into varying intensities of symptoms was made based on the similar treatment of scores in previous studies (Bener et al. 2011, Yusuf 2016, Sulaiman et al. 2017, Manoj et al. 2018, Jimenez-Zambrano et al. 2019).

Table 1. Description of study population by case and control

\begin{tabular}{|c|c|c|c|c|}
\hline Variables & Case - N (\%) & Control - N (\%) & Total - N (\%) & Statistics \\
\hline Age (years) & & & & $\chi^{2}=39.21, \mathrm{p}<0.001$ \\
\hline$\leq 37$ & $155(39.2)$ & $243(61.5)$ & $398(50.4)$ & \\
\hline$>37$ & $240(60.8)$ & $152(38.5)$ & $392(49.6)$ & \\
\hline Gender & & & & $\chi^{2}=0.00, p=1.00$ \\
\hline Male & $305(50.0)$ & $305(50.0)$ & $612(77.5)$ & \\
\hline Female & $90(50.0)$ & $90(50.0)$ & $178(22.5)$ & \\
\hline Residence & & & & $\chi^{2}=0.00, p=1.00$ \\
\hline Rural & 205 (51.9) & 205 (51.9) & $410(51.9)$ & \\
\hline Urban & $190(48.1)$ & $190(48.1)$ & $380(48.1)$ & \\
\hline Education & & & & $\chi^{2}=109.10, p<0.001$ \\
\hline Secondary & $25(6.3)$ & $12(3.0)$ & $37(4.7)$ & \\
\hline Higher secondary & $52(13.2)$ & $15(3.8)$ & $67(8.5)$ & \\
\hline Undergraduate & $190(48.1)$ & $95(24.1)$ & $285(36.1)$ & \\
\hline Masters or above & $128(32.4)$ & $273(69.1)$ & $401(50.8)$ & \\
\hline Family monthly income & & & & $\chi^{2}=5.58, p=0.02$ \\
\hline$\leq 40000 \mathrm{BDT}$ & $235(59.5)$ & $202(51.1)$ & $437(55.3)$ & \\
\hline$>40000 \mathrm{BDT}$ & $160(40.5)$ & $193(48.9)$ & $353(44.7)$ & \\
\hline Smoking & & & & $\chi^{2}=8.34, p=0.004$ \\
\hline Yes & $115(29.1)$ & $80(20.3)$ & $195(24.3)$ & \\
\hline No & $280(70.1)$ & $315(79.7)$ & $595(75.3)$ & \\
\hline Stress & & & & $\mathrm{t}=13.72, \mathrm{p}<0.001^{*}$ \\
\hline Mean \pm SD & $18.79 \pm 9.36$ & $10.07 \pm 8.49$ & $14.43 \pm 9.94$ & \\
\hline Normal & $104(26.3)$ & $224(56.7)$ & $328(41.5)$ & \\
\hline Mild & $74(18.7)$ & $114(28.9)$ & $188(23.8)$ & \\
\hline Moderate & $143(36.2)$ & $49(12.4)$ & $192(24.3)$ & $\mathrm{p}<0.001 * *$ \\
\hline Severe & $56(14.2)$ & $8(2.0)$ & $64(8.1)$ & \\
\hline Extremely severe & $18(4.6)$ & $0(0.0)$ & $18(2.3)$ & \\
\hline Anxiety & & & & $\mathrm{t}=13.487, \mathrm{p}<0.001^{*}$ \\
\hline Mean \pm SD & $11.04 \pm 9.37$ & $3.82 \pm 5.05$ & $7.43 \pm 8.34$ & \\
\hline Normal & $162(41.0)$ & $294(74.4)$ & $456(57.7)$ & \\
\hline Mild & $24(6.1)$ & $54(13.7)$ & $78(9.9)$ & \\
\hline Moderate & $80(20.3)$ & $28(7.1)$ & $108(13.7)$ & $\mathrm{p}<0.001 * *$ \\
\hline Severe & $45(11.4)$ & $12(3.0)$ & $57(7.2)$ & \\
\hline Extremely severe & $84(21.3)$ & $2(1.8)$ & $91(11.5)$ & \\
\hline Depression & & & & $\mathrm{t}=12.56, \mathrm{p}<0.001 *$ \\
\hline Mean \pm SD & $14.86 \pm 10.4$ & $6.75 \pm 7.51$ & $10.81 \pm 9.94$ & \\
\hline Normal & $122(28.4)$ & $272(68.9)$ & $384(48.6)$ & \\
\hline Mild & $62(25.7)$ & $32(8.1)$ & $94(11.9)$ & \\
\hline Moderate & $94(23.8)$ & $73(18.5)$ & $167(21.1)$ & $\mathrm{p}<0.001^{* *}$ \\
\hline Severe & $63(15.9)$ & $13(3.3)$ & $76(9.6)$ & \\
\hline Extremely severe & $64(16.2)$ & $5(1.3)$ & $69(8.7)$ & \\
\hline
\end{tabular}

$\chi^{2}=$ Chi square; *Independent Sample $\mathrm{t}$ test; **Fisher's exact test 
Important to note is that scores suggest the presence of depression, anxiety and stress, but are in no way diagnostic. Chi-square tests and Fisher's exact tests were conducted to examine the associations between covariates and symptoms of distress. In addition, an independent samples t-test was performed comparing the mean scores of mental health status between cases and controls. A conditional logistic regression was run to determine the relationship between having chronic diseases and distress symptom outcomes, while controlling for confounders. Matching was found to have not removed age as a confounder, thus age was controlled for during analysis (Pearce 2016). Data were analyzed using SAS (Statistical Analysis System) version 9.3. A p-value of less than 0.05 was considered significant. Odds ratios (OR) and their respective 95\% confidence intervals are provided to present results.

\section{RESULTS}

The mean age of cases was 38.37 years (Standard Deviation: 12.92), while that of the control group was 36.17 years (SD: 6.95). The total sample was split evenly (based on the median age - 37 years) between respondents older than 37 and those 37 and younger. However, matching for age was unsuccessful; there were more respondents older than 37 in the case group than the control. Inclusion of standard deviation ( \pm 5 years) during the matching process for age may have contributed to the large difference in age between the two groups. The majority of respondents were male, which constitutes a limitation of this study. The sample was split evenly between those who lived in rural areas and urban areas. Half of the respondents had a master's education or higher, and about a third had an undergraduate degree; very few respondents had only a secondary or higher secondary education. Monthly income of respondents was roughly equally distributed between $\leq 40000$ BDT (USD 470) and $>40000$ BDT. The most common chronic disease was asthma (27.6\%), followed by cardiovascular disease symptoms $(17.7 \%)$, and diabetes (15.9\%). Many (38.7\%) reported more than one disease or symptom. The overall prevalence of mild to extremely severe anxiety symptoms and depressive symptoms was $42.3 \%$ and $51.4 \%$ respecttively. The prevalence of mild to extreme stress levels was $58.5 \%$. The case group had prevalence estimates of distress symptoms that were $30-40 \%$ higher than the control. Cases also tended to be older and had slightly less post-secondary education than controls (Table 1).

About $74 \%$ of respondents with chronic diseases had mild or higher levels of stress compared to $43.3 \%$ of respondents without chronic diseases $(p<0.001)$. Fiftynine percent of respondents with chronic diseases had mild or higher levels of anxiety symptoms, whereas only $25.6 \%$ of respondents with no chronic diseases suffered from anxiety symptoms $(p<0.001)$. Of those with chronic diseases, $71.6 \%$ had mild to extremely severe levels of depressive symptoms, while only $31.1 \%$ of those without chronic diseases experienced depresssive symptoms $(\mathrm{p}<0.001)$. In the present study, the mean scores of stress $(\mathrm{t}=13.72, \mathrm{p}<0.001)$, anxiety $(\mathrm{t}=13.487$, $\mathrm{p}<0.001)$ and depressive symptoms $(\mathrm{t}=12.56, \mathrm{p}<0.001)$ were significantly higher in the case group compared to control group (Table 1).

Table 2. Chi-square tests of association between socio-demographic variables and mental health conditions of case group $(\mathrm{n}=395)$

\begin{tabular}{|c|c|c|c|c|c|c|c|}
\hline \multirow{2}{*}{ Variables } & \multicolumn{2}{|c|}{ Stress } & \multicolumn{2}{|c|}{ Anxiety } & \multicolumn{2}{|c|}{ Depression } & \multirow[t]{2}{*}{ Statistics } \\
\hline & No & Yes & No & Yes & No & Yes & \\
\hline \multicolumn{7}{|l|}{ Age (years) - n (\%) } & \multirow{3}{*}{$\begin{array}{l}\chi^{2}=0.002, p=0.965^{\mathrm{S}} \\
\chi^{2}=10.45, p=0.001^{\mathrm{A}} \\
\chi^{2}=4.186, p=0.041^{\mathrm{D}}\end{array}$} \\
\hline$\leq 37$ & $41(26.5)$ & $114(73.5)$ & $79(51.0)$ & $76(49.0)$ & $35(22.6)$ & $120(77.4)$ & \\
\hline$>37$ & $63(26.3)$ & $177(73.8)$ & $83(34.6)$ & $157(65.4)$ & $77(32.1)$ & $163(67.9)$ & \\
\hline \multicolumn{7}{|l|}{ Gender $-\mathrm{n}(\%)$} & \multirow{3}{*}{$\begin{array}{l}\chi^{2}=17.346, p<0.001^{\mathrm{S}} \\
\chi^{2}=29.494, \mathrm{p}<0.001^{\mathrm{A}} \\
\chi^{2}=8.633, \mathrm{p}=0.003^{\mathrm{D}}\end{array}$} \\
\hline Male & $96(31.3)$ & $211(68.7)$ & $148(48.2)$ & $159(51.8)$ & $98(31.9)$ & $209(68.1)$ & \\
\hline Female & $8(9.1)$ & $80(90.9)$ & $14(15.9)$ & $74(84.1)$ & $14(15.9)$ & $74(84.1)$ & \\
\hline \multicolumn{7}{|l|}{ Residence $-\mathrm{n}(\%)$} & \multirow{3}{*}{$\begin{array}{l}\chi^{2}=1.866, p=0.172^{\mathrm{S}} \\
\chi^{2}=12.007, p=0.001^{\mathrm{A}} \\
\chi^{2}=8.273, p=0.004^{\mathrm{D}}\end{array}$} \\
\hline Rural & $48(23.4)$ & $157(76.6)$ & $101(49.3)$ & $104(50.7)$ & $71(34.6)$ & $134(65.4)$ & \\
\hline Urban & $56(29.5)$ & $134(70.5)$ & $61(32.1)$ & $129(67.9)$ & $41(21.6)$ & $149(78.4)$ & \\
\hline \multicolumn{8}{|l|}{ Education - n (\%) } \\
\hline Secondary & $6(24.0)$ & $19(76.0)$ & $5(20.0)$ & $20(80.0)$ & $9(36.0)$ & $16(64.0)$ & \multirow{4}{*}{$\begin{array}{l}\chi^{2}=6.115, p=0.106^{\mathrm{S}} \\
\chi^{2}=48.192, p<0.001^{\mathrm{A}} \\
\chi^{2}=5.189, \mathrm{p}=0.158^{\mathrm{D}}\end{array}$} \\
\hline Higher secondary & $16(30.8)$ & $36(69.2)$ & $2(3.8)$ & $50(96.2)$ & $12(23.1)$ & $40(76.9)$ & \\
\hline Undergraduate & $58(30.5)$ & $132(69.5)$ & $82(43.2)$ & $108(56.8)$ & $62(32.6)$ & $128(67.4)$ & \\
\hline Master's and above & $24(18.8)$ & $104(81.3)$ & $73(57.0)$ & $55(43.0)$ & $29(22.7)$ & $99(77.3)$ & \\
\hline \multicolumn{7}{|c|}{ Family monthly income $-\mathrm{n}(\%)$} & \multirow{3}{*}{$\begin{array}{l}\chi^{2}=7.635, p=0.006^{S} \\
\chi^{2}=23.736, p<0.001^{\mathrm{A}} \\
\chi^{2}=16.079, p<0.001^{\mathrm{D}}\end{array}$} \\
\hline$\leq 40000 \mathrm{BDT}$ & $50(21.3)$ & $185(78.7)$ & $73(31.1)$ & $162(68.9)$ & $49(20.9)$ & $186(79.1)$ & \\
\hline$>40000 \mathrm{BDT}$ & $54(33.8)$ & $106(66.3)$ & $89(55.6)$ & $71(44.4)$ & $63(39.4)$ & $97(60.6)$ & \\
\hline \multicolumn{7}{|l|}{ Smoking status $-\mathrm{n}(\%)$} & \multirow{3}{*}{$\begin{array}{l}\chi^{2}=19.861, p<0.001^{S} \\
\chi^{2}=3.1132, p=0.078^{A} \\
\chi^{2}=1.899, p=0.168^{D}\end{array}$} \\
\hline Yes & $48(41.7)$ & $67(58.3)$ & $55(47.8)$ & $60(52.2)$ & $27(23.5)$ & $88(76.5)$ & \\
\hline No & $56(20.0)$ & $224(80.0)$ & $107(38.2)$ & $173(61.8)$ & $85(30.4)$ & $195(69.6)$ & \\
\hline
\end{tabular}

S - stress; A - anxiety; D - depression; $\chi^{2}=$ Chi-square 
Among cases, stressed respondents were significantly more likely than non-stressed respondents to be female $\left(\chi^{2}=17.346, \mathrm{p}<0.001\right)$, a member of lower wealth family $\left(\chi^{2}=7.635, \mathrm{p}=0.006\right)$ and a non-smoker $\left(\chi^{2}=19.861\right.$, $\mathrm{p}<0.001)$. Anxious respondents were also more likely than non-anxious respondents to be over 37 years old $\left(\chi^{2}=10.45\right.$, $\mathrm{p}=0.001)$, female $\left(\chi^{2}=29.494, \mathrm{p}<0.001\right)$, from urban areas $\left(\chi^{2}=12.007, \mathrm{p}=0.001\right)$, educated up to higher secondary $\left(\chi^{2}=48.192, \mathrm{p}<0.001\right)$ and from lower wealth family $\left(\chi^{2}=23.736, p<0.001\right)$. Depressed respondents were significantly more likely than non-depressed respondents to be below or equal to 37 years old $\left(\chi^{2}=4.186, p=0.041\right)$, female $\left(\chi^{2}=8.633, \mathrm{p}=0.003\right)$, from urban areas $\left(\chi^{2}=8.273, \mathrm{p}=0.004\right)$ and from lower wealth family $\left(\chi^{2}=16.079, \mathrm{p}<0.001\right)$ (Table 2).

Among controls, stressed respondents were significantly more likely than non-stressed respondents to be from urban areas $\left(\chi^{2}=6.712, p=0.010\right)$, educated at masters level $\left(\chi^{2}=47.235, \mathrm{p}<0.001\right)$, from a wealthy family $\left(\chi^{2}=12.564, \mathrm{p}<0.001\right)$ and a non-smoker $\left(\chi^{2}=8.640\right.$, $\mathrm{p}=0.003)$. Anxious respondents were also more likely than non-anxious respondents to be female $\left(\chi^{2}=21.819\right.$, $\mathrm{p}<0.001)$, from urban areas $\left(\chi^{2}=37.188, p<0.001\right)$, educated up to higher secondary $\left(\chi^{2}=11.101, \mathrm{p}=0.011\right)$ and from wealth family $\left(\chi^{2}=16.586, p<0.001\right)$. Depressed respondents were significantly more likely than non-depressed respondents to be from a wealthy family $\left(\chi^{2}=7.865\right.$, $\mathrm{p}=0.005)$ and a non-smoker $\left(\chi^{2}=5.805, \mathrm{p}=0.016\right)$ (Table 3).

The conditional logistic regression analysis presents socio-demographic factors and chronic diseases associated with distress symptoms after adjusting for confounders (Table 3). The matched variable age was also included in adjustment during analysis as age matching had not removed this as a confounding variable. Those with higher secondary (Odds Ratio [OR]: 0.20, Confidence Interval [CI]: $0.10-0.41$, and undergraduate (OR: 0.23, CI: 0.15-0.36) educations had lower odds of feeling stress than those with masters or higher degrees. Having any chronic disease increased the odds of having mild to extremely severe levels of stress (OR: 3.67, CI: 2.724.95). In particular, having asthma (OR: 4.43, CI: 2.617.53), diabetes (OR: 1.91, CI: 1.03-3.55), cardiovascular disease symptoms (OR: 6.70, CI: 3.32-13.50) and more than one disease (OR: 11.09, CI: 6.83-16.58) increased odds of experiencing mild to extremely severe levels of stress compared to healthy controls.

Respondents with secondary (OR: 7.04, CI: 2.7917.78) and higher secondary education (OR: 4.15, CI: 1.20-8.61) had higher adjusted odds of anxiety symptoms than master's degree holders. Respondents with any chronic disease also had increased odds of experiencing mild to extremely severe anxiety symptoms as compared to healthy controls (OR: 4.28, CI: 3.16-5.80). Having asthma (OR: $3.17, \mathrm{CI}: 1.85-5.42)$, diabetes (OR: 2.17, CI: 1.14-4.11), cardiovascular disease symptoms (OR: 8.67, CI: 5.54-14.57) and more than one disease (OR: 4.81, CI: 2.92-7.93) resulted in higher odds of experiencing anxiety symptoms compared to healthy controls.

Those with undergraduate degrees (OR: $0.48, \mathrm{CI}$ : $0.32-0.73$ ) had less odds of depressive symptoms than those with master's degrees or higher. Having any chronic disease (OR: 5.52, CI: 4.06-7.50), asthma (OR: 6.13, CI: 3.10-9.09), diabetes (OR: 3.40, CI: 1.83-6.31), cardiovascular disease symptoms (OR: 10.96, CI: 5.67-18.05) and more than one disease (OR: 9.61, CI: 5.74-13.09) increased the odds of experiencing mild or more severe depressive symptoms compared to healthy controls (Table 4).

Table 3. Chi-square tests of association between socio-demographic variables and mental health conditions of control group $(\mathrm{n}=395)$

\begin{tabular}{|c|c|c|c|c|c|c|c|}
\hline \multirow{2}{*}{ Variables } & \multicolumn{2}{|c|}{ Stress } & \multicolumn{2}{|c|}{ Anxiety } & \multicolumn{2}{|c|}{ Depression } & \multirow{2}{*}{ Statistics } \\
\hline & No & Yes & No & Yes & No & Yes & \\
\hline \multicolumn{7}{|l|}{ Age (years) - n (\%) } & \multirow{3}{*}{$\begin{array}{l}\chi^{2}=2.257, p=0.133^{\mathrm{S}} \\
\chi^{2}=0.552, p=0.458^{\mathrm{A}} \\
\chi^{2}=0.088, p=0.766^{\mathrm{D}}\end{array}$} \\
\hline$\leq 37$ & $145(59.7)$ & $98(40.3)$ & $184(75.7)$ & $59(24.3)$ & $166(68.3)$ & 77 (31.7) & \\
\hline$>37$ & $79(52.0)$ & $73(48.0)$ & $110(72.4)$ & $42(27.6)$ & $106(69.7)$ & $46(30.3)$ & \\
\hline \multicolumn{7}{|l|}{ Gender - n (\%) } & \multirow{3}{*}{$\begin{array}{l}\chi^{2}=0.000, p=0.993^{S} \\
\chi^{2}=21.819, p<0.001^{A} \\
\chi^{2}=0.614, p=0.433^{D}\end{array}$} \\
\hline Male & $173(56.7)$ & $132(43.3)$ & $244(80.0)$ & $61(20.0)$ & $207(67.9)$ & $98(32.1)$ & \\
\hline Female & $51(56.7)$ & $39(43.3)$ & $50(55.6)$ & $40(44.4)$ & $65(72.2)$ & $25(27.8)$ & \\
\hline \multicolumn{7}{|l|}{ Residence - n (\%) } & \multirow{3}{*}{$\begin{array}{l}\chi^{2}=6.712, p=0.010^{S} \\
\chi^{2}=37.188, p<0.001^{A} \\
\chi^{2}=0.696, p=0.404^{D}\end{array}$} \\
\hline Rural & $129(62.9)$ & $76(37.1)$ & $179(87.3)$ & $26(12.7)$ & $145(70.7)$ & $60(29.3)$ & \\
\hline Urban & $95(50.0)$ & $95(50.0)$ & $115(60.5)$ & $75(39.5)$ & $127(66.8)$ & $63(33.2)$ & \\
\hline \multicolumn{8}{|l|}{ Education $-\mathrm{n}(\%)$} \\
\hline Secondary & $8(66.7)$ & $4(33.3)$ & $4(33.3)$ & $8(66.7)$ & $8(66.7)$ & $4(33.3)$ & \multirow{4}{*}{$\begin{array}{l}\chi^{2}=47.235, p<0.001^{\mathrm{S}} \\
\chi^{2}=11.101, p=0.011^{\mathrm{A}} \\
\chi^{2}=5.752, p=0.124^{\mathrm{D}}\end{array}$} \\
\hline Higher secondary & $12(80.0)$ & $3(20.0)$ & $11(73.3)$ & $4(26.7)$ & $13(86.7)$ & $2(13.3)$ & \\
\hline Undergraduate & $80(84.2)$ & $15(15.8)$ & $73(76.8)$ & $22(23.2)$ & $72(75.8)$ & $23(24.2)$ & \\
\hline Master's and above & $124(45.4)$ & $149(54.6)$ & $206(75.5)$ & $67(24.5)$ & $179(65.6)$ & $94(34.4)$ & \\
\hline \multicolumn{7}{|c|}{ Family monthly income $-\mathrm{n}(\%)$} & \multirow{3}{*}{$\begin{array}{l}\chi^{2}=7.635, p=0.006^{\mathrm{S}} \\
\chi^{2}=23.736, p<0.001^{\mathrm{A}} \\
\chi^{2}=16.079, \mathrm{p}<0.001^{\mathrm{D}}\end{array}$} \\
\hline$\leq 40000 \mathrm{BDT}$ & $132(65.3)$ & $70(34.7)$ & $168(83.2)$ & $34(16.8)$ & $152(75.2)$ & $50(24.8)$ & \\
\hline$>40000 \mathrm{BDT}$ & $92(47.7)$ & $101(52.3)$ & $126(65.3)$ & $67(34.7)$ & $120(62.2)$ & $73(37.8)$ & \\
\hline \multicolumn{7}{|l|}{ Smoking status $-\mathrm{n}(\%)$} & \multirow{3}{*}{$\begin{array}{l}\chi^{2}=8.640, p=0.003^{\mathrm{S}} \\
\chi^{2}=1.635, \mathrm{p}=0.201^{\mathrm{A}} \\
\chi^{2}=5.805, \mathrm{p}=0.016^{\mathrm{D}}\end{array}$} \\
\hline Yes & $57(71.3)$ & $23(28.7)$ & $64(80.0)$ & $16(20.0)$ & $64(80.0)$ & $16(20.0)$ & \\
\hline No & $167(53.0)$ & $148(47.0)$ & $230(73.0)$ & $85(27.0)$ & $208(66.0)$ & $107(34.0)$ & \\
\hline
\end{tabular}

S - stress; A - anxiety; D - depression; $\chi^{2}=$ Chi-square 
Table 4. Conditional logistic regression analysis for socio-demographic and chronic disease factors associated with mental health outcomes

\begin{tabular}{|c|c|c|c|}
\hline \multirow{2}{*}{ Variables } & \multicolumn{3}{|c|}{ Odds Ratios (95\% Confidence Interval) } \\
\hline & Stress & Anxiety & Depression \\
\hline \multicolumn{4}{|l|}{ Age (ref.: $>37$ years) } \\
\hline$\leq 37$ years & $1.04(0.71-1.52)$ & $0.95(0.64-1.41)$ & $1.46(0.99-2.12)$ \\
\hline \multicolumn{4}{|c|}{ Education (ref.: masters and above ) } \\
\hline Secondary & $0.63(0.27-1.51)$ & $7.04(2.79-17.78)^{* *}$ & $0.71(0.31-1.64)$ \\
\hline Higher secondary & $0.20(0.10-0.41)^{*}$ & $4.15(1.20-8.61)^{* *}$ & $0.66(0.34-1.26)$ \\
\hline Undergraduate & $0.23(0.15-0.36)^{* *}$ & $1.37(0.92-2.05)$ & $0.48(0.32-0.73)^{* *}$ \\
\hline \multicolumn{4}{|c|}{ Family income (ref.: $>40000$ BDT) } \\
\hline$\leq 40000 \mathrm{BDTK}$ & $1.22(0.85-1.75)$ & $1.04(0.72-1.49)$ & $1.37(0.97-1.94)$ \\
\hline \multicolumn{4}{|l|}{ Chronic disease (ref.: no) } \\
\hline Yes & $3.67(2.72-4.95)^{* * *}$ & $4.28(3.16-5.80) * * *$ & $5.52(4.06-7.50)^{* * *}$ \\
\hline \multicolumn{4}{|c|}{ Types of diseases (ref.: no diseases) } \\
\hline Asthma & $4.43(2.61-7.53)^{* * *}$ & $3.17(1.85-5.42)^{* * *}$ & $6.13(3.10-9.09)^{* * *}$ \\
\hline Diabetes & $1.91(1.03-3.55)^{*}$ & $2.17(1.14-4.11)^{*}$ & $3.40(1.83-6.31)^{* * *}$ \\
\hline Cardiovascular symptoms & $6.70(3.32-13.50)^{* * *}$ & $8.67(5.54-14.57)^{* * *}$ & $10.96(5.67-18.05)^{* *}$ \\
\hline More than one diseases & $11.09(6.83-16.58)^{* * *}$ & $4.81(2.92-7.93)^{*}$ & $9.61(5.74-13.09) * * *$ \\
\hline
\end{tabular}

\section{DISCUSSION}

While a majority $(73.7 \%)$ of respondents with chronic diseases experienced mild to extremely severe levels of stress, $43.3 \%$ of healthy adults also experienced symptoms of stress. This prevalence of the healthy control group is higher than rates of healthy adult populations in other countries during the COVID-19 pandemic, such as the $32.1 \%$ reported in China (Wang et al. 2020) and $16.8 \%$ reported in the UK (Shevlin et al. 2020), but lower than the $64.3 \%$ reported in India (Kazmi et al. 2020).

Among diabetics, $49.2 \%$ experienced mild to extremely severe levels of stress, almost $150 \%$ more than healthy controls. This rate is higher than those reported in diabetic populations of Nigeria (Mosaku et al. 2008) and Saudi Arabia (AlKhathami et al. 2017), but similar to a rate reported in Mexico (55.10\%; Tovilla-Zarate et al. 2012). The prevalence estimate of depressive symptoms among diabetics was $55.6 \%$, which is again higher than rates reported in diabetic populations of Nigeria $(20 \%$; Mosaku et al. 2008) and Mexico (48\%; Tovilla-Zarate et al. 2012). This estimate was also higher than previous studies of depressive symptoms in Bangladeshi diabetics, which ranged from $34-45 \%$ (Roy et al. 2012, Islam et al. 2015a). This increase in depressive symptoms from prepandemic times may allude to negative impacts the COVID-19 pandemic has had on Bangladeshi people suffering from chronic illness. The Bangladeshi population's beliefs regarding disruptions of the health care system during the pandemic (Banna et al. 2020) and awareness of the heightened vulnerability of those with chronic diseases may contribute to this study's prevalence estimate of depression in diabetics (Onder et al. 2020, Ruan et al. 2020, Yang et al. 2020). However, this estimate is similar to the rate of depression in diabetics found in one study from Slovakia (53\%), a high-income country, which points to more factors driving depressive symptoms than just poor healthcare infrastructure (Majdan et al. 2012).
The mental health of those with existing comorbidity during the COVID-19 pandemic is rarely reported. Cases with more than one chronic disease had ten times the odds of reporting symptoms of stress, five times the odds of reporting symptoms of anxiety and eight times the odds of reporting symptoms of depression than healthy controls. Comorbidity of chronic diseases could be an important predictor or correlate of poor mental health and therefore could play a role in the detection of individuals with mental health disorders (Rozario \& Masho 2018).

This is the first study to report on the mental health outcomes of Bangladeshi adults suffering from chronic diseases during the COVID-19 pandemic. Matching cases with controls in this study limited bias in sampling. Limitations of this study include the limited research on this emerging topic, which hinders the understanding of this study's findings. All information was self-reported, which contributes to reporting bias. The DASS- 21 is not a diagnostic measure, and thus true mental health disorders cannot be ascribed to the symptoms. Recruitment methods only captured internet users, which reduces the generalizability of findings. The online nature of recruitment resulted in a more educated sample, which does not match the demographics of the entire country. The limited number of questions asked on this survey did not capture the complete living situations of respondents. For example, respondents were not asked whether they were spending the quarantine with relatives, roommates, alone, etc. Living situations with others may have an impact on the number of distress symptoms respondents experience and could be a confounding factor. Despite controlling for age as a confounder in analysis, the significant age difference between cases and controls, as well as the difference in smoking behavior, may contribute to the differences in distress symptom outcomes. Additionally, other confounding factors, such as domestic violence or exposure to stress-inducing media, were not captured in the survey. 


\section{CONCLUSIONS}

This study explores symptoms of distress in adults with chronic diseases during the COVID-19 pandemic. Individuals with underlying chronic physical health conditions are at greater risk of death from COVID-19, and thus were predicted to have higher rates of mental disorders than healthy individuals. Findings from this study suggest individuals with cardiovascular disease are most vulnerable to anxiety and depression symptoms during this time, and those with more than one chronic disease are most vulnerable to symptoms of stress. Having any of the chronic diseases included in this study or a combination of them predict high levels of mental health distress during the COVID-19 pandemic in Bangladesh. Health services and providers should take precautions with the mental health of their chronically ill patients by focusing on reaching out to this group via telemedicine sessions. Public health policymakers should incorporate mental health outreach into growing COVID-19 protocols, especially to high risk populations that have existing chronic disease.

\section{Acknowledgements:}

We thank our study respondents who participated in our survey.

\section{Ethical Approval:}

The Research Ethical Committee (REC) of the Department of Food Microbiology, Patuakhali Science and Technology University in Bangladesh reviewed and approved this study (Approval no.: FMB:24/04/2020: 02 ) by an expedited procedure. The research was carried out in accordance with the guidelines of the he Declaration of Helsinki in 1995 (as revised in Edinburgh 2000).

\section{Conflict of interest: None to declare.}

\section{Contribution of individual authors:}

Abu Sayeed: conceptualization, data curation, methodology, writing - original draft, validation;

Satyajit Kundu: conceptualization, data curation, writing - original draft, validation;

Md. Hasan Al Banna: conceptualization, writing - original draft; validation;

Enryka Christopher \& M. Tasdik Hasan: writing - review and editing, visualization, validation;

Musammet Rasheda Begum: formal analysis, software;

Sukanta Chowdhury: writing - review and editing, visualization;

Md Shafiqul Islam Khan: formal analysis, investigation, methodology, supervision, writing - review and editing, visualization, validation.

\section{References}

1. Alim SMAHM, Rabbani MG, Karim E, Mullick MSI, Mamun A Al \& Khan MZR: Assessment of depression, anxiety and stress among first year MBBS students of a public medical college, Bangladesh. Bangladesh $J$ Psychiatry 2015; 29:23-29

2. AlKhathami AD, Alamin MA, Alqahtani AM, Alsaeed WY, AlKhathami MA \& Al-Dhafeeri AH: Depression and anxiety among hypertensive and diabetic primary health care patients: Could patients' perception of their diseases control be used as a screening tool? Saudi Med J 2017; 38:621

3. Banna MH Al, Sayeed A, Kundu S, Christopher E, Hasan MT, Begum MR, Kormoker T, Dola STI, Hassan MM, Chowdhury S \& Khan MSI: The impact of the COVID-19 pandemic on the mental health of the adult population in Bangladesh: a nationwide cross-sectional study. Int $J$ Environ Health Res 2020; 1-12

4. Bener A, OAA Al-Hamaq A \& E Dafeeah E: High prevalence of depression, anxiety and stress symptoms among diabetes mellitus patients. Open Psychiatr J 2011; 5

5. Brooks SK, Webster RK, Smith LE, Woodland L, Wessely $S$, Greenberg $N$ \& Rubin GJ: The psychological impact of quarantine and how to reduce it: rapid review of the evidence. Lancet, 2020

6. Eysenbach G: Improving the quality of Web surveys: the Checklist for Reporting Results of Internet E-Surveys (CHERRIES). J Med Internet Res 2004; 6:e34

7. IEDCR: Covid-19 Status Bangladesh, 2020

8. Islam SMS, Ferrari U, Seissler J, Niessen L \& Lechner A: Association between depression and diabetes amongst adults in Bangladesh: a hospital based case-control study. $J$ Glob Health 2015a; 5

9. Islam SMS, Rawal LB \& Niessen LW: Prevalence of depression and its associated factors in patients with type 2 diabetes: a cross-sectional study in Dhaka, Bangladesh. Asian J Psychiatr 2015b; 17:36-41

10. Jakovljevic $M$, Bjedov S, Jaksic $N$ \& Jakovljevic I: COVID-19 pandemia and public and global mental health from the perspective of global health security. Psychiatr Danub 2020; 32:6-14

11. Jimenez-Zambrano J, Rodríguez-Díaz $R$ \& Acua-Chong $M G$ : Social anxiety among patients with multiple sclerosis: a population-based case-control study in Ecuador (P5. 2-099), 2019

12. Kazmi SSH, Hasan K, Talib S \& Saxena S: COVID-19 and Lockdwon: A Study on the Impact on Mental Health. Available SSRN 3577515, 2020

13. Khan AH, Sultana MS, Hossain S, Hasan MT, Ahmed HU, Sikder MT: The impact of COVID-19 pandemic on mental health \& wellbeing among home-quarantined Bangladeshi students: A cross-sectional pilot study. J Affect Disord 2020. doi:https://doi.org/10.1016/j.jad.2020.07.13

14. Lazzari C, Shoka A, Nusair A, \& Rabottini M: Psychiatry in Time of COVID-19 Pandemic. Psychiatr Danub 2020; 32:229-235

15. Le TA, Le MQT, Dang AD, Dang AK, Nguyen CT, Pham $H Q, V u$ GT, Hoang CL, Tran TT \& Vuong Q-H: Multilevel predictors of psychological problems among methadone maintenance treatment patients in difference types of settings in Vietnam. Subst Abuse Treat Prev Policy 2019; 14:39 
16. Lovibond PF \& Lovibond SH: The structure of negative emotional states: Comparison of the Depression Anxiety Stress Scales (DASS) with the Beck Depression and Anxiety Inventories. Behav Res Ther 1995; 33:335-343

17. Majdan M, Krajcovicova L, Pekarcikova J, Chereches $R$ \& O'Mullane M: Predictors of depression symptoms in patients with diabetes in Slovakia. Int J Psychiatry Med 2012; 44:351-366

18. Manoj MT, Joseph KA \& Vijayaraghavan G: Association of depression, anxiety, and stress with myocardial infarction: A case-control study. J Clin Prev Cardiol 2018; 7:86

19. Marčinko D, Jakovljević M, Jakšić N, Bjedov S\& Mindoljevic Drakulić A: The importance of psychodynamic approach during COVID-19 pandemic. Psychiatr Danub 2020; 32:15-21

20. McAlonan GM, Lee AM, Cheung V, Cheung C, Tsang $K W T$, Sham PC, Chua SE \& Wong JGWS: Immediate and sustained psychological impact of an emerging infectious disease outbreak on health care workers. Can J Psychiatry 2007; 52:241-247

21. Mosaku K, Kolawole B, Mume C \& Ikem R: Depression, anxiety and quality of life among diabetic patients: a comparative study. J Natl Med Assoc 2008; 100:73-78

22. Mukeshimana $M \&$ Chironda G: Depression and Associated Factors Among the Patients with Type 2 Diabetes in Rwanda. Ethiop J Health Sci 2019; 29

23. Onder $G$, Rezza $G$ \& Brusaferro $S$ : Case-fatality rate and characteristics of patients dying in relation to COVID-19 in Italy. Jama 2020; 323:1775-1776

24. Pearce N: Analysis of matched case-control studies. bmj 2016; 352:i969

25. Pellino G \& Spinelli A: How Coronavirus Disease 2019 Outbreak Is Impacting Colorectal Cancer Patients in Italy: A Long Shadow Beyond Infection. Dis Colon Rectum 2020; 63:720-722

26. Rahman M, Rahman MA, Flora MS \& Rakibuz-Zaman M: Depression and associated factors in diabetic patients attending an urban hospital of Bangladesh. Int J Collab Res Intern Med Public Heal 2011; 3:0

27. Rose $S$ \& Laan MJ Van der: Why match? Investigating matched case-control study designs with causal effect estimation. Int J Biostat 2009; 5

28. Roy T, Lloyd CE, Parvin M, Mohiuddin KGB \& Rahman M: Prevalence of co-morbid depression in out-patients with type 2 diabetes mellitus in Bangladesh. BMC Psychiatry 2012; 12:123

29. Rozario SS \& Masho SW: The Associations Between Mental Health Status, Hypertension, and Hospital Inpatient Visits in Women in the United States. Am J Hypertens 2018; 31:804-810

30. Ruan $Q$, Yang $K$, Wang $W$, Jiang $L \&$ Song J: Clinical predictors of mortality due to COVID-19 based on an analysis of data of 150 patients from Wuhan, China. Intensive Care Med 2020; 1-3

31. Sadiq MS, Morshed NM, Rahman W, Chowdhury NF, Arafat SMY \& Mullick MSI: Depression, Anxiety, Stress among Postgraduate Medical Residents: A Cross sectional Observation in Bangladesh. Iran J Psychiatry 2019; 14:192

32. Shevlin M, McBride O, Murphy J, Miller JG, Hartman TK, Levita L, Mason L, Martinez AP, McKay R \& Stocks TVA: Anxiety, Depression, Traumatic Stress, and COVID-19 Related Anxiety in the UK General Population During the COVID-19 Pandemic,. 2020

33. Sulaiman MAH, Al-Farsi YM, Al-Khaduri MM, Waly MI, Saleh $J$ \& Al-Adawi S: Psychological burden among women with polycystic ovarian syndrome in Oman: a case-control study. Int J Womens Health 2017; 9:897

34. Taber KS: The use of Cronbach's alpha when developing and reporting research instruments in science education. Res Sci Educ 2018; 48:1273-1296

35. Tovilla-Zarate C, Juarez-Rojop I, Jimenez YP, Jiménez $M A$, Vázquez $S$, Bermúdez-Ocaa D, Ramón-Frías $T$, Mendoza ADG, Garcia SP, \& Narváez LL: Prevalence of anxiety and depression among outpatients with type 2 diabetes in the Mexican population. PLoS One 2012; 7

36. Vandenbroucke JP, Elm E Von, Altman DG, Goetzsche $P C$, Mulrow CD, Pocock SJ, Poole C, Schlesselman JJ, Egger $M$ \& Initiative $S$ : Strengthening the Reporting of Observational Studies in Epidemiology (STROBE): explanation and elaboration. PLoS Med 2007; 4:e297

37. Wang C, Pan R, Wan X, Tan Y, Xu L, Ho CS, \& Ho RC: Immediate psychological responses and associated factors during the initial stage of the 2019 coronavirus disease (COVID-19) epidemic among the general population in China. Int J Environ Res Public Health 2020; 17:1729

38. WHO: WHO Director-General's opening remarks at the media briefing on COVID-19 - 11 March 2020, 2020 . Available at: https://www.who.int/dg/speeches/detail/whodirector-general-s-opening-remarks-at-the-mediabriefing-on-covid-19-11-march-2020

39. WHO: Coronavirus disease (COVID-19) Situation Report130, 2020b. Available at: https://www.who.int/docs/defaultsource/coronaviruse/situation-reports/20200529-covid19-sitrep-130.pdf?sfursn=bf7e7f0c_4

40. Williams JR: The Declaration of Helsinki and public health. Bull World Health Organ 2008; 86:650-652

41. Yang J, Zheng $Y$, Gou X, Pu K, Chen Z, Guo Q, Ji R, Wang H, Wang $Y$ \& Zhou Y: Prevalence of comorbidities and its effects in patients infected with SARS-CoV-2: a systematic review and meta-analysis. Int $J$ Infect Dis 2020; 94:91-95

42. Yusuf L: Depression, anxiety and stress among female patients of infertility; A case control study. Pakistan J Med Sci 2016; 32:1340

Correspondence:

Satyajit Kundu

Department of Biochemistry and Food Analysis, Patuakhali Science and Technology University

Patuakhali - 8602, Bangladesh

E-mail: satyajitnfs@gmail.com 